\title{
HDL cholesterol subclasses are associated with serum uric acid in Japanese men
}

\author{
Kengo MoriYama ${ }^{1}$
}

${ }^{1}$ Department of Clinical Health Science, Tokai University School of Medicine

\begin{abstract}
Objective Uric acid (UA) and high-density lipoprotein (HDL) subclasses are associated with inflammation, coronary heart disease, and metabolic syndrome (MetS). However, the relation between UA and HDL subclasses is not well understood.

Methods Subjects included 848 Japanese men not taking medication for hyperuricemia, hypertension, diabetes mellitus, dyslipidemia, or chronic renal disease; they underwent an annual health examination that included HDL subclass analyses.

Resufts When subjects were stratified by HDL2-C or HDL3-C levels, UA level decreased as HDL2-C level increased, while UA levels increased as HDL3-C levels increased. In a multiple linear regression analysis, age, waist circumference (WC), diastolic blood pressure (BP), logarithmic transformed triglyceride $\ln (\mathrm{TG})$ and HDL3 cholesterol (HDL3-C), were associated with UA level. In a multiple logistic regression analysis for upper tertile of UA ( $\geq 6.8 \mathrm{mg} / \mathrm{dL})$, WC, diastolic BP, In(TG), HDL2-C and HDL3-C were associated. Since this analysis indicated that MetS components were determinants of UA level along with HDL-C subclass, possible synergistic effects of HDL-C subclass and MetS components to determine UA level were assessed. A combination of the number of MetS components and stratification of HDL3-C affected UA levels; the mean UA level increased in subjects with increased MetS components and HDL3-C level.

Conclusion HDL-C subclasses were associated with UA level; particularly, a high HDL3-C level was associated with high UA level related to MetS in Japanese men. (HEP. 2018; 45: 563-568.)
\end{abstract}

Key words HDL2 cholesterol, HDL3 cholesterol, uric acid, metabolic syndrome

\section{INTRODUCTION}

Serum uric acid (UA) has been reported to be positively associated with the risk of atherosclerosis ${ }^{1}$, cardiovascular disease $(\mathrm{CVD})^{2)}$, hypertension ${ }^{3)}$, and metabolic syndrome (MetS) ${ }^{4)}$. Given that UA is associated with several conditions, this raised the possibility that UA might work synergistically with other risk factors during the development of atherosclerosis. In order to prevent the incidence of the above disorders, it is important to investigate the influence of individual risk factors and to understand the interrelationships of various risk factors with UA. Previous reports suggested a negative relationship between UA and high-density lipoprotein cholesterol (HDL-C) levels ${ }^{5,6}$, however, interactions between UA and HDL-C subclasses are not well understood.

HDL particles are heterogeneous with respect to density, size, composition, and surface charge. These particles can be separated into two main subfractions, HDL2 and HDL3, based on their density after ultracentrifugation ${ }^{7}$, and both have been reported to exhibit inverse associations with the incidence of $\mathrm{CVD}^{8)}$. We previously demonstrated the importance of analyzing levels of HDL-C subclasses, as well as overall HDL-C, when evaluating

Received: November 20, 2017, Accepted: January 24, 2018

${ }^{1}$ Address; Tokai University School of Medicine

1838 Ishikawa-machi, Hachioji, Tokyo 192-0032, Japan

TEL: +81-42-639-1111, FAX: +81-42-639-1178

E-mail: osaru3moving@gmail.com an individual's lifestyle habits ${ }^{9-11)}$, insulin resistance, and MetS ${ }^{12)}$. HDL2-C levels are associated with alcohol consumption, waist circumference (WC), smoking, and exercise. On the other hand, HDL3-C levels are only associated with alcohol consumption ${ }^{11}$. We reported that the HDL2-C/HDL3-C ratio was associated with MetS components, insulin resistance, and high-molecular-weight adiponectin (HMW-Ad) levels and was thus useful for evaluating MetS in Japanese individuals ${ }^{12)}$. We also reported that changes in HDL2-C/HDL3-C were inversely correlated with changes in WC, insulin resistance, and low-density lipoprotein cholesterol (LDLC), and positively correlated with HMW-Ad and good lifestyle habits ${ }^{13)}$. It is apparent that not only HDL-C, but also HDL subclasses and ratios should be investigated with respect to coronary heart disease (CHD) risk, obesity, MetS, and lifestyle habits.

Therefore, it is important to investigate possible connections between serum HDL-C subclasses and UA level to protect against atherosclerotic events and MetS. This study aimed to clarify the relationship between HDL-C subclasses and UA in subjects not receiving treatment for hyperuricemia, hypertension, dyslipidemia, diabetes mellitus, or chronic renal disease.

\section{SUBJECTS AND METHODS}

\section{Subjects}

A total of 1,282 subjects underwent annual health examinations, including HDL-C subclass analyses, at the Health Evaluation and Promotion Center, Tokai University Hachioji Hospital, between April 2011 and March 2016. After excluding 439 sub- 
jects who were taking medication for hyperuricemia, hypertension, diabetes mellitus, dyslipidemia, or chronic renal disease, 848 subjects were ultimately included in this study. Medical histories were obtained using self-administered questionnaires and interviews conducted by nurses.

\section{Measurements}

WC was measured at the level of the umbilicus while the subject was standing and during slight expiration. Blood pressure (BP) was measured on the upper right arm using an automatic BP monitor (TM-2655P; A\&D, Tokyo, Japan) while the subject was seated. Blood samples were collected in tubes coated with heparin early in the morning after an overnight fast. UA levels were measured using the uricase- $N$-(3-sulfopropyl)-3-methoxy5-methylaniline method with a Wako L-Type UA M kit (Wako Pure Chemicals, Osaka, Japan). Hyperuricemia was defined as plasma UA levels $>7.0 \mathrm{mg} / \mathrm{dL}^{14)}$. Fasting plasma glucose (FPG) was measured using the hexokinase glucose 6-phosphate dehydrogenase method with a Wako L-type Glu 2 kit (Wako Pure Chemicals, Osaka, Japan). Fasting immunoreactive insulin (FIRI) levels were measured using a fluorescence enzyme immunoassay (ST AIA-PACK IRI; Toso, Tokyo, Japan). Serum high-sensitivity C-reactive protein (hsCRP) levels were measured using latex agglutination turbidimetry. Aspartate aminotransferase (AST) and alanine aminotransferase (ALT) were measured using JSCC transferable method with L-Type AST.J2 and L-Type ALT.J2 (Wako Pure Chemicals, Osaka, Japan). LDL-C was calculated using the Friedewald formula ${ }^{15}$. HDL-C and triglyceride (TG) levels were measured using visible spectrophotometry (Determiner L HDL-C, and Determiner L TG II, respectively; Kyowa Medex, Tokyo, Japan). HDL2-C and HDL3-C levels were determined via ultracentrifugation. Briefly, after plasma was centrifuged using an L-60 centrifuge (Beckman Coulter, Brea, USA) at $22,300 \times g$ for $4 \mathrm{~h}$ at a plasma density of $1.063 \mathrm{~kg} / \mathrm{L}$ and a solvent density of $1.125 \mathrm{~kg} / \mathrm{L}$, adjusted by adding solid $\mathrm{KBr}, 40 \%$ volume from the top was aspirated, yielding HDL (a) and HDL3 (b) fractions. The cholesterol concentration of each fraction was measured, and HDL2-C was calculated as follows: [(a) $-(b) \times$ $1.54] \times 0.6^{16)}$.

Verbal consent for the analytical use of anonymized health records was obtained from all subjects. The study protocol was approved by the institutional ethics committee of the Tokai University School of Medicine.

\section{Definitions of MetS}

A diagnosis of MetS requires any three of the following five factors: central obesity, as determined by WC ( $\geq 85 \mathrm{~cm}$ for men, $\geq 90 \mathrm{~cm}$ for women); increased FPG levels ( $>5.5 \mathrm{mmol} / \mathrm{L} ; 100$ $\mathrm{mg} / \mathrm{dL}$ ); increased TG levels ( $\geq 1.7 \mathrm{mmol} / \mathrm{L} ; 150 \mathrm{mg} / \mathrm{dL}$ ); low HDL-C levels ( $<1.0 \mathrm{mmol} / \mathrm{L} ; 40 \mathrm{mg} / \mathrm{dL}$ in males and $<1.3$ $\mathrm{mmol} / \mathrm{L} ; 50 \mathrm{mg} / \mathrm{dL}$ in females), or an elevated BP [systolic BP $\geq$ $130 \mathrm{mmHg}$ or diastolic $\mathrm{BP} \geq 85 \mathrm{mmHg}]^{17,18)}$.

\section{Statistical analysis}

Due to skewed distributions of TG and hsCRP, logarithmic transformation was applied. Scheffe's multiple comparisons test was used to compare mean values across more than two groups. The relationships between HDL-C subclasses and UA were investigated using Pearson's correlation coefficient. A multiple linear regression analysis was performed to identify significant determinants of UA. Age, BMI, WC, systolic BP, diastolic BP, FPG, FIRI, LDL-C, HDL2-C, HDL3-C, logarithmic transformed TG $(\ln (\mathrm{TG})), \ln (\mathrm{hsCRP})$, AST, ALT, exercise habit, amount of alcohol consumption, and current smoking status were used as independent variables. A multiple logistic regression analysis to calculate odds ratios (ORs) for the hyperuricemia (UA $>7.0 \mathrm{mg}$ / $\mathrm{dL}$ ) was performed. The same variables listed for the multiple linear regression analyses were used for this analysis. Subjects were classified as smoker or non-smoker. Additionally, those who exercised for $\geq 30 \mathrm{~min} /$ day more than twice per week were classified as habitual exercisers. Alcohol consumption was surveyed by asking how many units of sake were consumed in a day, where 1 unit $(180 \mathrm{~mL})$ was taken to be the equivalent to $25 \mathrm{~g}$ of alcohol. A stepwise procedure was used to select variables for the multiple linear and logistic regression analyses. Statistical analyses were performed using SAS software, version 9.4 (SAS Institute Inc., Cary, NC, USA). All $P$-values were two-tailed, and a $P$-value $<0.05$ was considered statistically significant.

\section{RESULTS}

Table 1 lists the subjects' characteristics. The mean HDL-C, HDL2-C, HDL3-C, and UA levels for the total subjects were 59.0 $\mathrm{mg} / \mathrm{dL}, 36.6 \mathrm{mg} / \mathrm{dL}, 21.5 \mathrm{mg} / \mathrm{dL}$, and $6.3 \mathrm{mg} / \mathrm{dL}$, respectively, and the prevalence of hyperuricemia was $29.5 \%$. The characteristics of the study subjects according to the tertile value of UA level (T1: $<5.8 \mathrm{mg} / \mathrm{dL}$ ), T2: 5.8 to $<6.8 \mathrm{mg} / \mathrm{dL}, \mathrm{T} 3: \geq 6.8 \mathrm{mg} /$ $\mathrm{dL}$ ) are also shown in Table 1. The upper tertile of UA was often accompanied by the worst metabolic profile (BMI, WC, BP, FIRI, TG, HDL-C, AST, ALT and number of MetS components). HDL2-C level of the middle and upper tertiles of UA were significantly lower than the lower tertile. HDL3-C level of the upper tertile of UA were significantly higher than the lower tertile. Although no significant differences were observed, LDL-C levels gradually increased as UA levels increased.

Pearson's correlation coefficient was used to assess the univariate associations between HDL-C subclasses and UA. UA levels exhibited a positive correlation with HDL3-C ( $r=0.110$, confidence interval (CI): $0.043-0.176, P=0.0013)$, but showed negative correlations with HDL-C and HDL2-C $(r=-0.131, \mathrm{CI}$ : $-0.196--0.064, \mathrm{P}=0.0001$ and $r=-0.164$, CI: $-0.229-$ $-0.098, P<0.0001$, for each).

To test the relationship between HDL subclasses and UA, mean UA levels were compared when subjects were stratified by tertile for either HDL2-C (Fig. 1 (a)) or HDL3-C levels (Fig. 1 (b)). UA levels decreased as HDL2-C increased. UA levels in the middle and upper tertiles of HDL2-C were significantly higher than the lower tertile. On the contrary, UA levels increased as HDL3-C increased. UA levels in the upper tertile of HDL3-C were significantly higher than the lower tertile.

Next, determinants of UA level were analyzed by multiple linear regression analysis (Table 2). Among the following variables, age, BMI, WC, systolic BP, diastolic BP, FPG, FIRI, LDLC, HDL2-C, HDL3-C, $\ln (\mathrm{TG}), \ln (\mathrm{hsCRP}), \mathrm{AST}$, ALT, exercise 
Table 1 Characteristics of study subjects

\begin{tabular}{|c|c|c|c|c|}
\hline Variables & $\begin{array}{c}\text { Total } \\
\mathrm{n}=848\end{array}$ & $\begin{array}{c}\mathrm{T} 1(<5.8 \mathrm{mg} / \mathrm{dL}) \\
\mathrm{n}=282\end{array}$ & $\begin{array}{c}\mathrm{T} 2(5.8-<6.8 \mathrm{mg} / \mathrm{dL}) \\
\mathrm{n}=266\end{array}$ & $\begin{array}{c}\mathrm{T} 3(\geq 6.8 \mathrm{mg} / \mathrm{dL}) \\
\mathrm{n}=300\end{array}$ \\
\hline Age (years) & $53.2 \pm 12.3$ & $54.8 \pm 12.9$ & $52.8 \pm 12.4$ & $52.0 \pm 11.6^{*}$ \\
\hline BMI $\left(\mathrm{kg} / \mathrm{m}^{2}\right)$ & $23.9 \pm 3.3$ & $23.0 \pm 3.1$ & $23.9 \pm 3.1^{\text {** }}$ & $24.8 \pm 3.6^{\star \star, \# \#}$ \\
\hline Waist circumference $(\mathrm{cm})$ & $84.5 \pm 9.2$ & $81.9 \pm 8.8$ & $84.4 \pm 8.3^{* *}$ & $87.1 \pm 9.5^{\star \star, \# \#}$ \\
\hline Systolic BP (mmHg) & $122.5 \pm 17.3$ & $119.7 \pm 16.9$ & $123.0 \pm 16.4$ & $124.6 \pm 18.1^{* *}$ \\
\hline Diastolic BP (mmHg) & $78.0 \pm 13.0$ & $75.4 \pm 13.0$ & $78.0 \pm 11.9$ & $80.4 \pm 13.3^{* *}$ \\
\hline FPG (mg/dL) & $103.2 \pm 19.5$ & $103.0 \pm 18.2$ & $102.2 \pm 13.4$ & $104.1 \pm 24.5$ \\
\hline $\mathrm{FIRI}(\mu \mathrm{IU} / \mathrm{mL})$ & $6.50 \pm 4.93$ & $5.49 \pm 3.91$ & $6.46 \pm 5.12$ & $7.49 \pm 5.42^{\star \star, \#}$ \\
\hline TG (mg/dL) & $128.9 \pm 99.5$ & $106.6 \pm 64.7$ & $128.9 \pm 86.4^{*}$ & $149.7 \pm 128.9^{* \star, \#}$ \\
\hline HDL-C (mg/dL) & $59.0 \pm 14.6$ & $61.3 \pm 14.7$ & $58.4 \pm 14.2$ & $57.2 \pm 14.5^{\star *}$ \\
\hline HDL2-C (mg/dL) & $36.6 \pm 12.3$ & $39.0 \pm 12.8$ & $36.4 \pm 11.8^{*}$ & $34.5 \pm 11.9^{* *}$ \\
\hline HDL3-C (mg/dL) & $21.5 \pm 3.5$ & $21.1 \pm 3.4$ & $21.4 \pm 3.3$ & $21.9 \pm 3.7^{*}$ \\
\hline LDL-C (mg/dL) & $122.9 \pm 31.8$ & $119.9 \pm 29.1$ & $123.3 \pm 31.6$ & $125.5 \pm 34.1$ \\
\hline $\mathrm{UA}(\mathrm{mg} / \mathrm{dL})$ & $6.3 \pm 1.2$ & $5.0 \pm 0.6$ & $6.2 \pm 0.3^{\star *}$ & $7.6 \pm 0.7^{* *, \# \#}$ \\
\hline hsCRP (mg/dL) & $0.118 \pm 0.300$ & $0.109 \pm 0.279$ & $0.129 \pm 0.400$ & $0.116 \pm 0.203$ \\
\hline AST (IU/L) & $24.0 \pm 12.3$ & $23.2 \pm 15.4$ & $22.9 \pm 7.5$ & $25.7 \pm 12.2^{\#}$ \\
\hline ALT (IU/L) & $27.5 \pm 21.7$ & $24.4 \pm 22.2$ & $27.2 \pm 19.3$ & $30.7 \pm 22.9^{* *}$ \\
\hline Numbers of MetS & $1.3 \pm 1.2$ & $1.0 \pm 1.1$ & $1.3 \pm 1.1$ & $1.7 \pm 1.2^{* *, \ldots \#}$ \\
\hline
\end{tabular}

Variables are given as means \pm standard deviations. Subjects were stratified into four groups according to UA level. T1; lower tertile,T2; middle tertile,T3; upper tertile of UA in each group. BMI, body mass index; BP, blood pressure; FPG, fasting plasma glucose; FIRI, fasting immunoreactive insulin; TG, triglyceride; HDL-C, high-density lipoprotein cholesterol; HDL2-C, high-density lipoprotein 2 cholesterol; HDL3-C, high-density lipoprotein 3 cholesterol; LDL-C, low-density lipoprotein cholesterol calculated by Friedewald formula; UA, uric acid; hsCRP, high sensitivity C-reactive protein; AST, aspartate transaminase; ALT, alanine transferase

${ }^{* *} p<0.01,{ }^{*} p<0.05$ (T1 vs T2, T1 vs T3), ${ }^{\#} p<0.01,{ }^{*} p<0.05$ (T2 vs T3) by Scheffe's multiple comparison test.

(a)

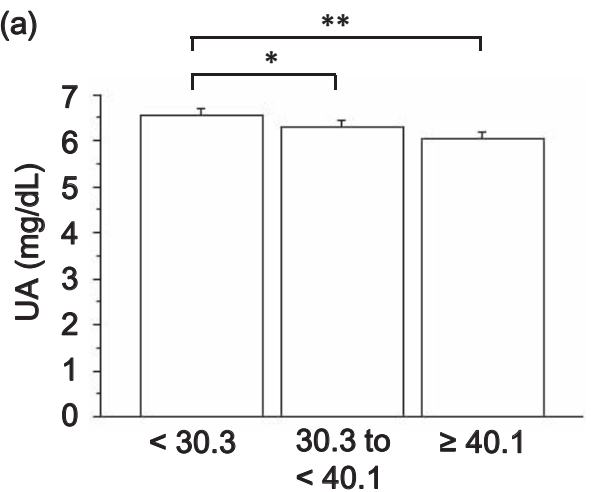

$\mathrm{HDL} 2-\mathrm{C}(\mathrm{mg} / \mathrm{dL})$ (b)

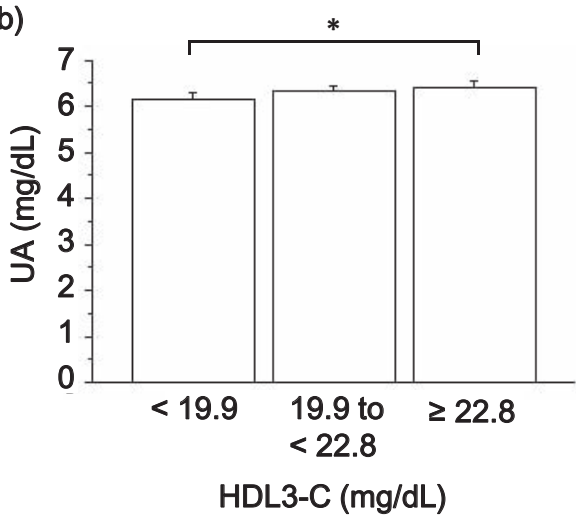

Fig. 1 Bar graph of mean UA values with $95 \%$ confidence intervals after stratifying subjects according to HDL2-C (a) and HDL3-C (b) levels.

$* P<0.05, * * P<0.01$ by Scheffe's multiple comparison test.

UA, uric acid; HDL2-C, high-density lipoprotein 2 cholesterol; HDL3-C, high-density lipoprotein 3 cholesterol

habit, amount of alcohol consumption, and current smoking status, six variables (age, $\mathrm{WC}$, diastolic $\mathrm{BP}, \ln (\mathrm{TG}), \ln (\mathrm{hsCRP})$, and HDL3-C) were selected by a stepwise procedure. Standardized regression coefficients in the multiple regression analysis were higher for $\mathrm{WC}, \ln (\mathrm{TG})$, and HDL3-C than for diastolic BP, $\ln (\mathrm{hsCRP})$ and age. Thus, WC, $\ln (\mathrm{TG})$, and HDL3-C were mainly and positively associated with UA levels.

Determinants of hyperuricemia (UA $>7.0 \mathrm{mg} / \mathrm{dL}$ ) were analyzed by multiple logistic regression analysis (Table 3). Five variables (WC, diastolic BP, $\ln (\mathrm{TG})$, HDL2-C, and HDL3-C) were selected by a stepwise procedure. The OR for $\ln (\mathrm{TG})$ was the highest and HDL3-C was second among the selected variables, indicating that $\ln (\mathrm{TG})$ and HDL3-C was dominantly associated with hyperuricemia.

According to the multiple linear regression for UA and multiple logistic regression analysis for the hyperuricemia, selected variables were components of MetS (WC, diastolic BP and $\ln (\mathrm{TG})$ ). Accordingly, the association of UA and HDL3-C levels 
Table 2 Multiple liner regression analysis for UA

\begin{tabular}{lcccr}
\hline & \multicolumn{4}{c}{ Multiple liner regression } \\
\cline { 2 - 5 } & RC & SRC & \multicolumn{1}{c}{$t$} & \multicolumn{1}{c}{$P$} \\
\hline Age & -0.0096 & -0.09605 & -2.89 & 0.0039 \\
WC & 0.02101 & 0.15608 & 4.25 & $<0.0001$ \\
Diastolic BP & 0.01096 & 0.11506 & 3.36 & 0.0008 \\
Ln(TG) & 0.37412 & 0.16391 & 4.52 & $<0.0001$ \\
Ln(hsCRP) & 0.07482 & 0.07027 & 2.04 & 0.0417 \\
HDL3-C & 0.05336 & 0.15235 & 4.60 & $<0.0001$ \\
\hline
\end{tabular}

Variable selection was made by a stepwise procedure. RC, regression coefficient; SRC, standardized regression coefficient; WC, waist circumference; BP, blood pressure; In(TG), logarithmic transformed triglyceride; In(hsCRP), logarithmic transformed high sensitivity C-reactive protein; HDL3-C, high-density lipoprotein 3 cholesterol
Table 3 Multiple logistic regression analysis for upper tertile of UA

\begin{tabular}{lccccc}
\hline & \multicolumn{5}{c}{ Multiple logistic regression } \\
\cline { 2 - 6 } & RC & SE & OR & $95 \% \mathrm{Cl}$ & $P$ \\
\hline WC & 0.0337 & 0.00969 & 1.034 & $1.015-1.054$ & 0.0005 \\
Diastolic BP & 0.0194 & 0.00637 & 1.020 & $1.007-1.032$ & 0.0024 \\
Ln(TG) & 0.3626 & 0.1678 & 1.437 & $1.034-1.997$ & 0.0307 \\
HDL2-C & -0.0176 & 0.00820 & 0.983 & $0.967-0.998$ & 0.0319 \\
HDL3-C & 0.0854 & 0.0238 & 1.089 & $1.040-1.141$ & 0.0003 \\
\hline
\end{tabular}

Variable selection was made by a stepwise procedure. $\mathrm{RC}$, regression coefficient; SE, standard error; OR, odds ratio; CL, confidence interval; WC, waist circumference; $\mathrm{BP}$, blood pressure; $\ln (\mathrm{TG})$, logarithmic transformed triglyceride; HDL2-C, high-density lipoprotein 2 cholesterol; HDL3-C, high-density lipoprotein 3 cholesterol (a)

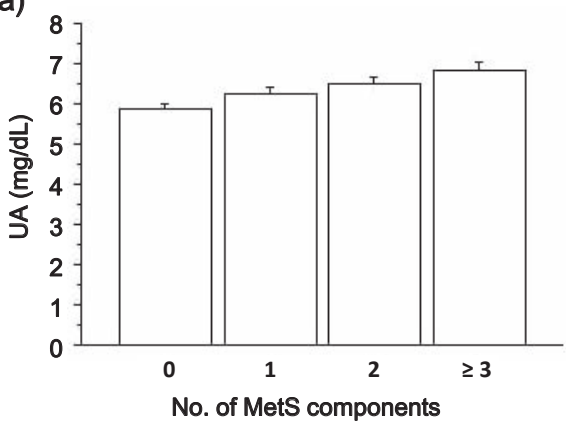

(b)

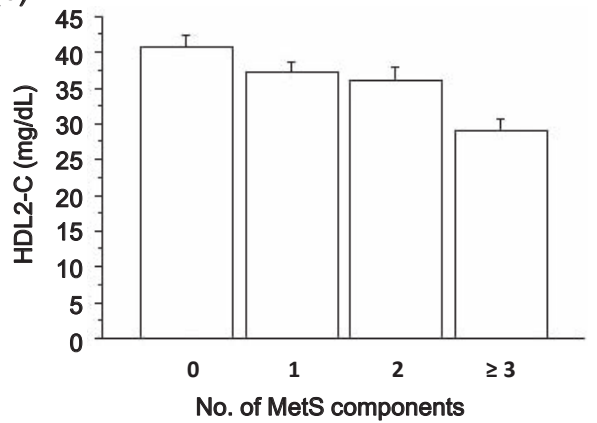

(c)

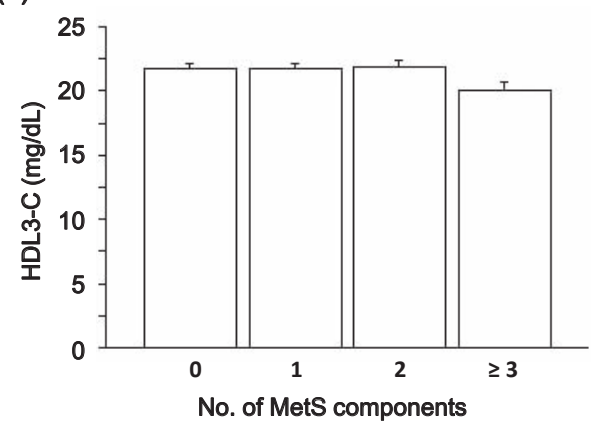

(d)

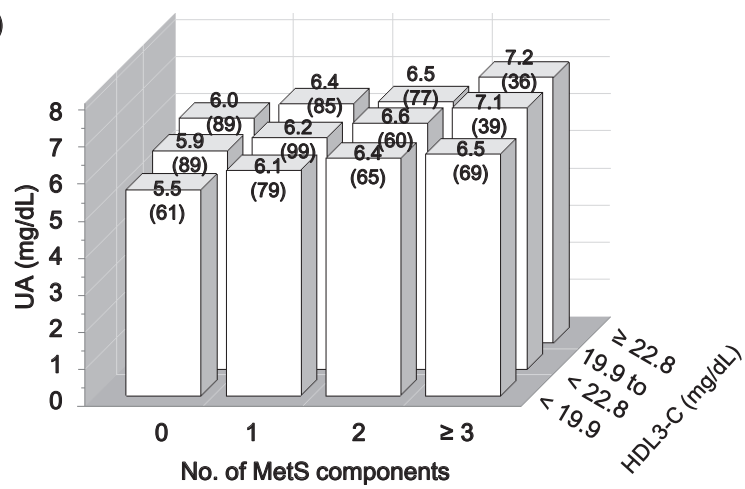

Fig. 2 (a) Bar graph of mean UA values with $95 \%$ confidence intervals after stratifying subjects according to number of MetS components. (b) Bar graph of mean UA values after stratifying subjects according to number of MetS components and HDL3-C levels.

Numbers on the bars represent mean UA values (mg/dL) (upper) and number of subjects (lower) of each group.

UA, uric acid; MetS, metabolic syndrome; HDL2-C, high-density lipoprotein 2 cholesterol; HDL3-C, high-density lipoprotein 3 cholesterol

in relation to MetS was investigated (Fig. 2). In agreement with previous reports ${ }^{19,20)}$, mean UA levels gradually increased as the number of MetS components $(0,1,2$, and $\geq 3)$ in the four groups increased (Fig. 2 (a)). Mean HDL2-C levels gradually decreased as the number of MetS components $(0,1,2$, and $\geq 3)$ in the four groups increased (Fig. 2 (b)). On the other hand, it was not clear whether HDL3-C levels were associated with the number of MetS components, since the mean HDL3-C levels stratified by number of MetS components were imbalanced (Fig. 2 (c)). However, when the combined effects of HDL3-C and number of MetS components to UA levels were investigated, mean UA levels generally increased with increasing number of MetS components and HDL3-C levels (Fig. 2 (d)).

\section{DISCUSSION}

The present study showed that HDL-C subclasses were associated with UA; in particular, high HDL3-C levels were associated with high UA levels in relationship to MetS in Japanese men. To the best of my knowledge, this is the first report to describe the association of HDL3-C and UA levels relative to MetS compo- 
nents.

In agreement with previous study which showed higher UA was strong predictor of small, dense HDL particles in middleaged European subjects ${ }^{21)}$, HDL3-C was associated with UA level in the current study. Small, dense HDL particles by gradient gel electrophoresis may be compatible, at least in part, with HDL3 determined by ultracentrifugation. As UA level positively associated with makers of inflammation, the authors speculated that oxidation stress related to the small HDL particles could result in compensatory elevation of $\mathrm{UA}^{21}$. The reason why marker of inflammation $(\ln (\mathrm{hsCRP}))$ was not correlated with hyperuricemia in the current study could be partly because of the differences in the clinical characteristics of study subjects; those in the previous study had a higher frequency of CHD risk which is often accompanied by inflammation, while subjects in the current study were healthier. On the other hand, mean serum UA level was much higher in the current study ( 6.3 versus $4.8 \mathrm{mg} / \mathrm{dL}$ ), partly due to the selection of the current study subjects where women were not studied.

Previous study indicated that serum UA was negatively related to large HDL in Chinese subjects, since multiple liner regression analysis indicated that large HDL was selected ${ }^{6}$. This result was in agreement with the current study, since HDL2-C was selected in multiple logistic regression analysis (Table 3). In addition, subjects with high UA level in the current study were accompanied with high CVD risk factors including dyslipidemia and MetS. However, I reported a controversial result showing that serum UA was positively related to small HDL-C (HDL3-C), since multiple linear regression analysis and multiple logistic regression analysis indicated that HDL3-C was determinant for UA (Table 2 and 3). It is reported that when subjects were stratified by quintile value of serum UA level, large and intermediate HDL-C decreased, while the trend of small HDL-C was imbalanced ${ }^{6}$.

Visceral obesity and insulin resistance contribute to postprandial hypertriglyceridemia and low HDL-C, both of which are components of MetS. These conditions ultimately stimulate hepatic TG output ${ }^{22)}$. It is widely accepted that serum UA closely related to $\mathrm{MetS}^{4}$. Moreover, we previously reported that HDL-C and HDL2-C showed very strong positive correlation, and HDL2$\mathrm{C}$ levels were decreased by obesity ${ }^{11)}$. Therefore, it is reasonable to see the subjects with highest UA concomitant with higher BMI, WC, TG showed lower HDL-C and HDL2-C.

Since both UA and HDL appeared to be associated with CVD, inflammation, and MetS, they may share similar characteristics. UA has antioxidant effects in physiological environments or in association with antioxidants ${ }^{23}$. In vitro, UA has an antioxidant effect on native LDL as well as a prooxidant effect on mildly oxidized LDL ${ }^{24)}$. However, despite the potential antioxidant effect of UA, numerous studies have revealed positive associations between serum UA concentration and various disorders, most of which are components of MetS. HDL protects LDL from oxidative damage by free radicals and therefore exerts antioxidative and anti-inflammatory properties ${ }^{25}$. Circulating HDL particles, particularly small, dense, protein-rich HDL3, may provide potent protection of LDL in vivo from oxidative damage by free radicals in the arterial intima, resulting in inhibited generation of proinflammatory oxidized lipids ${ }^{25}$. It has yet to be established whether different levels of uric acid may directly modify the lipid prolife, including HDL.

It is unclear how HDL3 modify the association between UA and MetS components. It is well recognized that oxidative stress increased in MetS, low physical activity, and smoking ${ }^{17)}$. Under chronic inflammation status, such as in active condition of rheumatoid arthritis, HDL-C levels decrease and the composition of HDL changes ${ }^{26)}$. UA contributes to the overall inflammatory state of subjects. Unexpectedly, the results revealed association between hsCRP and UA by multiple linear regression, not in multiple logistic regression analysis. This is probably due to the characteristics of the study subjects, as none received medication for hyperuricemia, hypertension, diabetes mellitus, dyslipidemia, or chronic renal disease, all of which are associated with inflammation. Indeed, mean hsCRP level $(0.118 \mathrm{mg} / \mathrm{dL})$ in the current study was much lower than previously reported ${ }^{21)}$. Therefore, it is probable that under milder oxidative stress where inflammation marker did not reflect the stress, HDL3 might protect against such stress, resulted in compensatory increased HDL3 level.

The association between serum UA and CVD has long been recognized. However, it has not been definitively established whether serum UA is merely a marker for risk or a causative agent in CVD. Many studies have found that serum UA independently associated with CVD risk and events ${ }^{27,28)}$. On the other hand, compelling arguments have also been reported that the apparent association is primarily resulted from the strong collinear interaction of serum UA and established CVD risk factors ${ }^{29,30)}$. Some of the variation in the study results likely relates to differences in population characteristics and statistical methods.

The limitations of our study include that it was cross-sectional in nature, which prevented the establishment of a causal relationship. The subjects in this study were middle-aged Japanese men; therefore, it is possible that the relationship between UA levels and clinical markers were affected by gender, and ethnicity. Finally, the results were calculated from the data of only a fraction of the subjects who underwent annual health examinations; therefore, the findings might not be generalizable to all Japanese men.

In conclusion, both HDL2-C and HDL3-C were associated with serum UA levels in subjects who are not receiving treatment for hyperuricemia, hypertension, diabetes mellitus, dyslipidemia, or chronic renal disease. In particular, high HDL3-C was associated with a high UA in relationship to MetS in Japanese men.

The authors state that they have no Conflict of Interest (COI).

\section{REFERENCES}

1) Ishizaka N, Ishizaka Y, Toda E, Hashimoto H, Nagai R, Yamakado M. Higher serum uric acid is associated with increased arterial stiffness in Japanese individuals. Atherosclerosis 2007; 192: 1317. 
2) Chen JH, Chuang SY, Chen HJ, Yeh WT, Pan WH. Serum uric acid level as an independent risk factor for all-cause, cardiovascular, and ischemic stroke mortality: a Chinese cohort study. Arthritis Rheum 2009; 61: 225-32.

3) Forman JP, Choi H, Curhan GC. Uric acid and insulin sensitivity and risk of incident hypertension. Arch Intern Med 2009; 169: 155-62.

4) Sui X, Church TS, Meriwether RA, Lobelo F, Blair SN. Uric acid and the development of metabolic syndrome in women and men. Metabolism 2008; 57: 845-52.

5) Teng F, Zhu R, Zou C, Xue Y, Yang M, Song H, et al. Interaction between serum uric acid and triglycerides in relation to blood pressure. J Hum Hypertens 2011; 25: 686-91.

6) Zhang Y, Xu RX, Li S, Zhu CG, Guo YL, Sun J, et al. Lipoprotein subfractions partly mediate the association between serum uric acid and coronary artery disease. Clin Chim Acta 2015; 441: 10914.

7) Glomset JA. The plasma lecithin: cholesterol acyltransferase reaction. J Lipid Res 1968; 9: 155-67.

8) Sweetnam PM, Bolton CH, Yarnell JW, Bainton D, Baker IA, Elwood PC, et al. Associations of the HDL2 and HDL3 cholesterol subfractions with the development of ischemic heart disease in British men: the Caerphilly and Speedwell Collaborative Heart Disease Studies. Circulation 1994; 90: 769-74.

9) Hiratsuka N, Yamada C, Mitsuhashi T, Inabe F, Araida N, Takahashi E. Significance of high HDL cholesterol levels in Japanese men with metabolic syndrome. Intern Med 2011; 50: 2113-20.

10) Moriyama K, Takahashi E, Negami M, Otsuka H, Mitsuhashi T, Tsurugano S, et al. Evaluation of high-density lipoprotein cholesterol levels in Japanese women. Tokai J Exp Clin Med 2012; 37: 77-83.

11) Moriyama K, Takahashi E. Relationships of high-density lipoprotein 2 and 3 cholesterols with lifestyle habit factors in Japanese adults. Ningen Dock Int 2014; 1: 54-62.

12) Moriyama $\mathrm{K}$, Negami $\mathrm{M}$, Takahashi E. $\mathrm{HDL}_{2}$-cholesterol/HDL $\mathrm{HL}_{3}$ cholesterol ratio was associated with insulin resistance, highmolecular weight adiponectin, and components for metabolic syndrome in Japanese. Diabetes Res Clin Pr 2014; 106: 360-5.

13) Moriyama K, Takahashi E. HDL2/HDL3 ratio changes, metabolic syndrome markers, and other factors in a Japanese population. J Atheroscler Thromb 2016; 23: 704-12.

14) Japanese Society of Gout and Nucleic Acid Metabolism. Guideline for the Management of Hyperuricemia and Gout. Tokyo, Japan: Japanese Society of Gout and Nucleic Acid Metabolism 2012 (in Japanese).

15) Friedewald WT, Levy RI, Fredrickson DS. Estimation of the concentration of low-density lipoprotein cholesterol in plasma, without use of the preparative ultracentrifuge. Clin Chem 1972; 18: 499-502.

16) Bronzert TJ, Brewer HB Jr. New micro method for measuring cholesterol in plasma lipoprotein fractions. Clin Chem 1977; 23: 2089-98.
17) Teramoto T, Sasaki J, Ueshima H, Egusa G, Kinoshita M, Shimamoto K, et al. Metabolic Syndrome. J Atheroscler Tromb 2008; 15: 1-5.

18) Alberti KG, Eckel RH, Grundy SM, Zimmet PZ, Cleeman JI, Donato KA, et al. International Diabetes Federation Task Force on Epidemiology and Prevention; National Heart, Lung, and Blood Institute; American Heart Association; World Heart Federation; International Atherosclerosis Society; International Association for the Study of Obesity. Harmonizing the metabolic syndrome: a joint interim statement of the International Diabetes Federation Task Force on Epidemiology and Prevention; National Heart, Lung, and Blood Institute; American Heart Association; World Heart Federation; International Atherosclerosis Society; and International Association for the Study of Obesity. Circulation 2009; 120: 1640-5.

19) Puig JG, Martínez MA. Hyperuricemia, gout and the metabolic syndrome. Curr Opin Rheumatol 2008; 20: 187-91.

20) Stibůrková B, Pavlíková M, Sokolová J, Kožich V. Metabolic syndrome, alcohol consumption and genetic factors are associated with serum uric acid concentration. PLoS One 2014; 9: e97646.

21) Vekic J, Jelic-Ivanovic Z, Spasojevic-Kalimanovska V, Memon L, Zeljkovic A, Bogavac-Stanojevic N, et al. High serum uric acid and low-grade inflammation are associated with smaller LDL and HDL particles. Atherosclerosis 2009; 203: 236-42.

22) Johnson RJ, Stenvinkel P, Martin SL, Jani A, Sánchez-Lozada LG, Hill JO, et al. Redefining metabolic syndrome as a fat storage condition based on studies of comparative physiology. Obesity 2013; 21: 659-64.

23) Gersch C, Palii SP, Kim KM, Angerhofer A, Johnson RJ, Henderson GN. Inactivation of nitric oxide by uric acid. Nucleosides Nucleotides Nucleic Acids 2008; 27: 967-78.

24) Patterson RA, Horsley ET, Leake DS. Prooxidant and antioxidant properties of human serum ultrafiltrates toward LDL: important role of uric acid. J Lipid Res 2003; 44: 512-21.

25) Navab M, Ananthramaiah GM, Reddy ST, Van Lenten BJ, Ansell BJ, Fonarow GC, et al. The oxidation hypothesis of atherogenesis: the role of oxidized phospholipids and HDL. J Lipid Res 2004; 45: 993-1007.

26) Feingold KR, Grunfeld C. Effect of inflammation on HDL structure and function. Curr Opin Lipidol 2016; 27: 521-30.

27) Fang J, Alderman MH. Serum uric acid and cardiovascular mortality the NHANES I epidemiologic follow-up study, 1971-1992. National Health and Nutrition Examination Survey. JAMA 2000; 283: $2404-10$

28) Niskanen LK, Laaksonen DE, Nyyssönen K, Alfthan G, Lakka HM, Lakka TA, et al. Uric acid level as a risk factor for cardiovascular and all-cause mortality in middle-aged men: a prospective cohort study. Arch Intern Med 2004; 164: 1546-51.

29) Culleton BF, Larson MG, Kannel WB, Levy D. Serum uric acid and risk for cardiovascular disease and death: the Framingham Heart Study. Ann Intern Med 1999; 131: 7-13.

30) Dobson A. Is raised serum uric acid a cause of cardiovascular disease or death? Lancet 1999; 354: 1578. 